\title{
Análisis de la frecuencia cardiaca con relación a las acciones de juego de un arquero escolar en competencia
}

\author{
Behavior of the heart rate and its relation with the goalkeeper's play actions during the competition
}

\section{Resumen}

\author{
Andrés Castellanos Medina \\ Óscar Bernal" \\ Orlando Sánchez
}

\begin{abstract}
La estadistica siempre ha experimentado un extraordinario auge como disciplina de aplicación en el deporte; en el fútbol ha estado orientada a la técnica y a la preparación física de los jugadores y casi nunca a la del arquero. El siguiente trabajo es un estudio de campo que tiene como objetivo analizar el comportamiento de la frecuencia cardiaca $(\mathrm{FC})$ en infantiles arqueros ante las acciones de juego, para establecer parámetros para la dosificación en el entrenamiento de infantiles escolares. Se filmaron los movimientos que realizó el arquero y se tomaron las frecuencias cardiacas en le momento de la acción y a los cinco segundos ( $5^{\prime \prime}$ ) siguientes por medio de un monitor cardiaco (marca polar); durante cuatro partidos de competencia realizados en la primera ronda clasificatoria del torneo Uncoli (Unión de Colegios Internacionales) de fútbol, categoria infantil masculino, en la ciudad de Bogotá. Para establecer la relación entre la FC y las acciones de juego se pasaron los datos a hojas de Excel en la que se agruparon en cuatro categorias (1. Acciones defensivas. 2. Acciones compuestas. 3. Acciones ofensivas, y 4. Habilidades); posteriormente se realizaron las gráficas que nos permitieron interpretar el comportamiento de la FC frente a las acciones de juego.
\end{abstract}

Palabras clave: Arquero, frecuencia cardiaca, análisis, acciones de juego, FC máxima, FC minima.

\section{Abstract}

Key words: $\quad$ Archer, heart frequency, analysis, game stocks, maxim FC, FC minimum.

Fecha de recepción: 21 de octubre de 2005.

Fecha de aceptación: 11 de noviembre de 2005.

Licenciado en Educación Física de la Universidad Pedagógica Nacional. Entrenador de arqueros de la selección Bogotá, 1999, 2000, 2001 y 2002. Entrenador de arqueros del Club Ecopetrol 2004, docente del Colegio Los Nogales y entrenador de arqueros de la Escuela de Fútbol la Academia.

** Especialista en Pedagogía del Entrenamiento Deportivo, docente Universidad Pedagógica Nacional., entrenador profesional.

*** Biólogo y químico de la Universidad Pedagógica Nacional. Médico de la Universidad Nacional de Colombia. Especialista en Pedagogía del Entrenamiento Deportivo. Docente de la Universidad Pedagógica Nacional y de la Universidad Nacional. Profesor de fisiología de la Facultad de Educación Fisica de la Universidad Pedagógica Nacional. 
$\mathrm{El}$ arquero recibe la importancia que le damos, pero sólo tiene la que le dedicamos.

\section{Introducción}

Es de gran interés el estudio de la frecuencia cardiaca (FC) como una variable fisiológica que brinda información en el entrenamiento deportivo, ya que su control permite conocer los cambios que ocurren en el organismo como producto de los esfuerzos físicos de diferente potencia y duración; este indicador en el fútbol en particular ha sido tema de investigación de los especialistas, quienes se han dirigido tanto al control de la carga en el entrenamiento como al estudio de los esfuerzos en el partido, permitiendo conocer en mayor medida las demandas energéticas que ocurren en el juego e incrementar la exactitud de la dosificación de las cargas físicas (Lanza, 2003).

En la actualidad la competencia es una parte esencial del entrenamiento, y se considera la forma de comprobación más completa, ya que en ella se hallan de forma clara y precisa las posibles variables del rendimiento. La planeación de un modelo de entrenamiento en deportes de equipo demanda un análisis de las exigencias que presenta la competencia. Por tanto, el control del entrenamiento requiere la utilización de sistemas de registro y análisis que permitan controlar las variables que interactúan en el rendimiento (Gutiérrez y cols., citado por Valdivielso, 1996).

Desde el punto de vista fisiológico, uno de los aspectos más importantes y útiles para los entrenadores y preparadores físicos para evaluar la condición física de los jugadores de fútbol, $y$ a la cual se ha recurrido como parámetro esencial de la parte fisiológica para esta investigación, es la frecuencia cardiaca. Teniendo en cuenta esta referencia, se estudió la frecuencia cardiaca de un arquero de fútbol en competencia de categoría infantil, con relación a las acciones de juego predominantes del torneo. Uncoli.

El arquero jugó 296 minutos en cuatro partidos, de los cuales se registraron $3.552 \mathrm{FC}$ en el monitor polar, en valores absolutos. La FC máxima en los cuatro partidos es 204,3 lpm (latidos por minuto), la cual se presentó en el primer partido. La FC mínima es de 111 lpm y se presentó en el tercer y cuarto partidos. La FC máxima media obtenida es $186,4 \mathrm{lpm}$, la FC mínima media es $107,5 \mathrm{lpm}$ y la FC promedio es 152,2 lpm.

Intervino en 154 acciones de juego, distribuidas así: acciones defensivas, 59; acciones compuestas, 37 , acciones ofensivas, 34 y habilidades, 24 .
Luego se analizaron las gráficas por partido y por acción para observar el comportamiento de la FC.

\section{Marco referencial}

La frecuencia cardiaca es el número de latidos ventriculares por minuto, contados en los registros del electrocardiograma o en las curvas de presión sanguínea. Se puede determinar también la frecuencia cardiaca por auscultación o por palpación sobre el corazón tanto en reposo como durante el ejercicio. El gasto cardiaco es el producto de la frecuencia cardiaca por el volumen sistólico (volumen de sangre eyectada por el ventrículo durante la sístole), y corresponde al volumen cardiaco o débito cardiaco $(\mathrm{Q}=$ volumen sistólico $\mathrm{x}$ frecuencia cardiaca), el cual puede aumentar durante la actividad física a valores entre 20 y 30 litros/minuto. El consumo de oxígeno es el volumen de oxígeno $\left(\mathrm{a} 0^{\circ} \mathrm{C}, 760 \mathrm{mmHg}\right.$, seco $=$ STPD) extraído del aire inspirado, habitualmente expresado en litros por minuto ( $\mathrm{VO}_{2} \mathrm{ml} / \mathrm{kg} / \mathrm{min}$ ). Otra relación de la frecuencia cardiaca FC corresponde al pulso de oxígeno, es decir, el consumo de oxígeno reposo/FC y su relación con el peso corporal (Astrad y Rodahl, 1985).

La frecuencia cardiaca del recién nacido es rápida y está sujeta a amplias fluctuaciones. Por lo general oscila entre 120 y 140 latidos por minuto, pero puede aumentar hasta 170 o más durante los períodos de llanto y actividad, y descender a 70-90 durante el sueño. Conforme crece el niño, el pulso se hace más lento.

\begin{tabular}{|c|c|c|c|c|c|c|}
\hline Edad & \multicolumn{2}{|c|}{$\begin{array}{l}\text { Limites inferiores de } \\
\text { la normalidad }\end{array}$} & \multicolumn{2}{|c|}{ Promedio } & \multicolumn{2}{|c|}{$\begin{array}{l}\text { Limites superiores de } \\
\text { la normalidad }\end{array}$} \\
\hline Recién nacido & \multicolumn{2}{|c|}{80} & \multicolumn{2}{|c|}{120} & \multicolumn{2}{|c|}{170} \\
\hline 1 a 11 meses & \multicolumn{2}{|c|}{80} & \multicolumn{2}{|c|}{120.} & \multicolumn{2}{|c|}{160} \\
\hline 2 años & \multicolumn{2}{|c|}{80} & \multicolumn{2}{|c|}{110} & \multicolumn{2}{|c|}{130} \\
\hline 4 años & \multicolumn{2}{|c|}{80} & \multicolumn{2}{|c|}{100} & \multicolumn{2}{|c|}{120} \\
\hline 6 años & \multicolumn{2}{|c|}{75} & \multicolumn{2}{|c|}{100} & \multicolumn{2}{|c|}{115} \\
\hline 8 años & \multicolumn{2}{|c|}{70} & \multicolumn{2}{|c|}{90} & \multicolumn{2}{|c|}{110} \\
\hline \multirow[t]{2}{*}{10 años } & \multicolumn{2}{|c|}{70} & \multicolumn{2}{|c|}{90} & \multicolumn{2}{|c|}{110} \\
\hline & Niñas & Niños & Niñas & Niños & Niñas & Niños \\
\hline 12 años & 70 & 65 & 90 & 85 & 110 & 105 \\
\hline 14 años & 65 & 60 & 85 & 80 & 105 & 100 \\
\hline 16 años: & 60 & 55 & 80 & 75 & 100 & 95 \\
\hline 18 años & 55 & 50 & 75 & 70 & 95 & 90 \\
\hline
\end{tabular}

Tabla 1. Indica la frecuencia cardiaca inferior, promedio y superior en las diversas edades, según, observaciones de varios autores (Vaughan, 2002).

La frecuencia cardiaca máxima teórica según Fox, se determina en individuos sanos restando la edad en años a 220 (Fox, 1989) o a 235 en deportistas de rendimiento (Dirix, 1988). 
Para Sierra (1998), sin duda la frecuencia cardiaca es el indicador de esfuerzo mas empleado. Debido a esto se ha utilizado en gran cantidad de test y protocolos de esfuerzo para estimar y monitorear la intensidad del ejercicio (Eston y Williams, 1988)

En los niños la University of Motor Sciences (IUSM) de roma, (Capranica, 2001), realizó un estudio en partidos de competencia de niños de 11 años llamado Heart rate and match análisis in pre-pubescent soccer players en el que se realizó un seguimiento con video y monitor cardiaco (observando cada 5 segundos la FC), en partidos de competencia. Se llegó a la conclusión que en los partidos la frecuencia cardiaca no excedía los $170 \mathrm{lpm}$ en un $84 \%$ del tiempo.

En el fútbol se ha estudiado la frecuencia cardiaca orientada a los jugadores de campo. Lanza (2003) encontró valores medios individuales de frecuencia cardiaca en el partido en un rango entre 160 y $170 \mathrm{lpm}$; el valor medio hallado en cinco jugadores fue de 166,29 $\pm 3,25$ $\mathrm{lpm}$. Al conocer que la frecuencia cardiaca en jugadores de primera división se enmarca entre 165 y 175 lpm, los estudios anteriores se realizaron en jugadores de campo.

Pirnay (1993) en sus estudios encontró resultados medios de $167 \pm 4 \mathrm{lpm}$. Valores más altos presentó Boeda (185), quien situó la frecuencia cardiaca media durante el partido alrededor de los $170 \mathrm{lpm}$, planteando que coincide con el $85 \%$ de la frecuencia cardiaca máxima, mientras que para Ekblon (1990) fueron ligeramente superiores con un resultado de $175 \mathrm{lpm}$.

No obstante, en la actualidad no se han encontrado registros del arquero, debido a la falta de literatura específica relacionada con la frecuencia cardiaca del arquero en competencia. El presente estudio de caso surge con el propósito de registrar y analizar el comportamiento de la frecuencia cardiaca en relación con las acciones de juego que realiza un arquero escolar en competencia.

\section{Objetivos}

a. Determinar el comportamiento de la frecuencia cardiaca ante las acciones de juego asociadas al esfuerzo físico que se realiza durante un partido de competencia en el torneo Uncoli en un arquero decategoría infantil de 14 años.

b. Especificar las diferencias en le esfuerzo físicoque el arquero presenta en durante el partido.

c. Aportar pruebas científicas que generen una guía para la planificación y la elaboración de programas de preparación física de arqueros acorde con la competencia.

\section{Material y método}

La investigación es un estudio de caso realizado en la temporada de Uncoli de la categoría infantil del año 2002-2003 escolar para calendario B en la ciudad de Bogotá, analizando a un arquero durante cuatro partidos de la primera ronda clasificatoria. Estos partidos se jugaban en dos tiempos de 35 minutos, con un descanso intermedio de 12 a 15 minutos. El sistema que se utilizó fue el de grabar los partidos con una videograbadora Sony Handycam Vision video HI8, y colocarle un monitor cardiaco marca polar Advantage Plus, el cual grabaría los cambios de la frecuencia cardiaca 12 veces por minuto (cada cinco segundos).

Para recoger los registros de la frecuencia cardiaca se utilizó el monitor polar Advantage Plus, el cual se configuró para registrar la FC cada cinco segundos y se le colocó al arquero antes de iniciar el partido. Cuando el juez daba la orden para iniciar el partido el arquero accionaba el botón para iniciar el registro en el polar.

La obtención de los registros se llevó acabo con el software del polar y se pasaron a Excel para realizar las gráficas y sus promedios.

\section{Resultados y análisis}

Primero se establecen las frecuencias cardiacas de los cuatro partidos y se obtienen los siguientes datos: la FC máxima promedio, 186,42 lpm, la FC mínima promedio, 107,58 lpm y FC promedio fue de 152,26 lpm (gráfica 1).

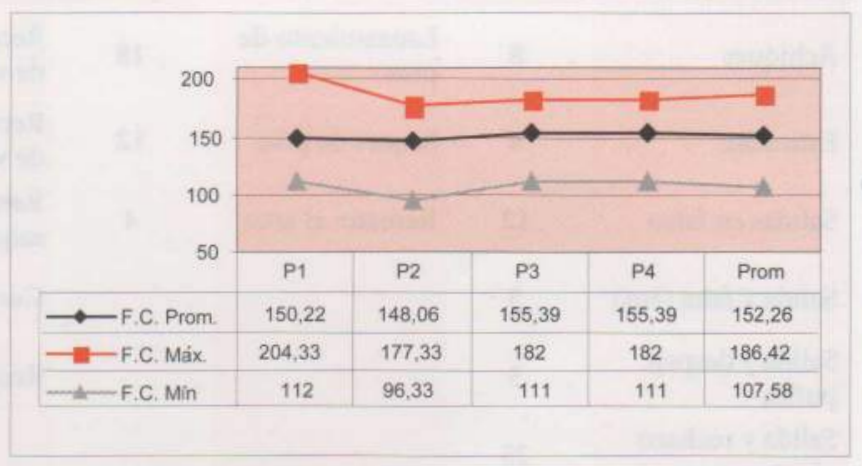

Gráfica 1. Frecuencia de los partidos de fútbol

Estos datos aportan información referente a la intensidad compet itiva y a la carga soportada por el arquero en un partido. 
Así mismo, analizamos por medio del video las acciones de juego que el arquero realizó durante los cuatro partidos y su relación con la frecuencia cardiaca de cada movimiento en dos momentos durante la acción (F1) y a los cinco segundos después de realizado el movimiento (F2).
Se observaron 154 acciones durante los cuatro partidos (296 minutos de juego): 77 en los primeros tiempos y 77 en los segundos tiempos. El promedio fue de 38,25 acciones por partido. Un promedio de 37 minutos por tiempo y 74 minutos por partido (tabla 2).

\begin{tabular}{|c|c|c|c|c|c|c|c|c|c|}
\hline \multirow{2}{*}{$\begin{array}{l}\text { Partido } \\
\text { Tiempo }\end{array}$} & \multicolumn{2}{|c|}{ Partido 1} & \multicolumn{2}{|c|}{ Partido 2} & \multicolumn{2}{|c|}{ Partido 3} & \multicolumn{2}{|c|}{ Partido 4} & \multirow[b]{2}{*}{$\begin{array}{c}\text { Total } \\
\text { acciones }\end{array}$} \\
\hline & Tiempo & $\begin{array}{c}\text { No. } \\
\text { acciones }\end{array}$ & Tiempo & $\begin{array}{c}\text { No. } \\
\text { acciones }\end{array}$ & Tiempo & $\begin{array}{c}\text { No. } \\
\text { acciones }\end{array}$ & Tiempo & $\begin{array}{c}\text { No. } \\
\text { acciones }\end{array}$ & \\
\hline $\begin{array}{l}\text { Primer } \\
\text { tiempo }\end{array}$ & $37^{\prime} 27^{\prime \prime}$ & 25 & $36^{\prime} 22^{\prime \prime}$ & 14 & $35^{\prime} 25^{\prime \prime}$ & 18 & $35^{\prime} 44^{\prime \prime}$ & 20 & 77 \\
\hline $\begin{array}{l}\text { Segundo } \\
\text { tiempo }\end{array}$ & $38^{\prime} 50^{\prime \prime}$ & 24 & $37^{\prime} 25^{\prime \prime}$ & 16 & $36^{\prime} 20^{\prime \prime}$ & 16 & $38^{\prime} 28^{\prime \prime}$ & 21 & 77 \\
\hline Total & $76 ' 17^{\prime \prime}$ & 49 & $73^{\prime} 47^{\prime \prime}$ & 30 & $71^{\prime} 45^{\prime \prime}$ & 34 & $74^{\prime} 12^{\prime \prime}$ & 41 & 154 \\
\hline
\end{tabular}

Tabla 2. Acciones de los cuatro partidos de fútbol

Estas 154 acciones se agruparon en cuatro categorías, así: 1 . acciones defensivas, $59(38 \%) ; 2$. acciones ofensi- vas, 34 (22\%); 3. acciones compuestas, 37 (24\%); 4 . habilidades, 24 (16\%); (Gráfica 2 y tabla 3 ).

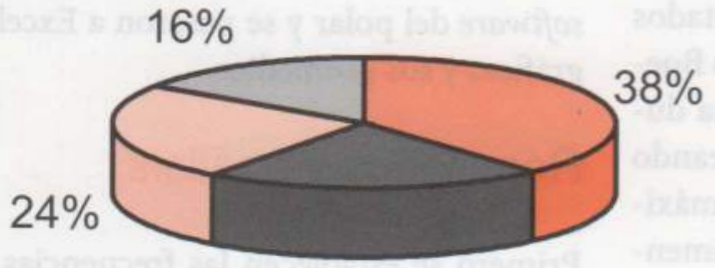

$22 \%$
Acciones defensivas

Acciones ofensivas

Acciones compuestas

Habilidades

Gráfica 2. Acciones de juego

\begin{tabular}{|c|c|c|c|c|c|c|c|}
\hline Defensivas & $\begin{array}{l}\text { No. } \\
\text { Acciones }\end{array}$ & Ofensivas & $\begin{array}{l}\text { No. } \\
\text { Acciones }\end{array}$ & Compuestas & $\begin{array}{l}\text { No. } \\
\text { Acciones }\end{array}$ & Habilidades & $\begin{array}{l}\text { No. } \\
\text { Acciones }\end{array}$ \\
\hline Achiques & 8 & $\begin{array}{l}\text { Lanzamiento de } \\
\text { piso }\end{array}$ & 18 & $\begin{array}{l}\text { Recepción media y saque } \\
\text { de volea }\end{array}$ & 10 & $\begin{array}{l}\text { Desplazamientos } \\
\text { laterales }\end{array}$ & 8 \\
\hline Estiradas & 4 & Saques de piso & 12 & $\begin{array}{l}\text { Recepción baja y saque } \\
\text { de volea }\end{array}$ & 8 & Salto vertical & 2 \\
\hline Salidas en falso & 12 & Remates al arco & 4 & $\begin{array}{l}\text { Recepción con caída y } \\
\text { saque }\end{array}$ & 5 & Estático & 13 \\
\hline Salida y falta (ent) & 3 & 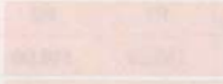 & & Control pie y pase & 9 & $\begin{array}{l}\text { Desplazamiento } \\
\text { frontal }\end{array}$ & 1 \\
\hline $\begin{array}{l}\text { Salida y despeje } \\
\text { puños }\end{array}$ & 5 & $x^{2}$ & & Recepción alta y saque & 5 & 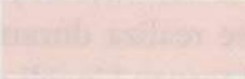 & $x_{2}$ \\
\hline $\begin{array}{l}\text { Salida y rechazo } \\
\text { pie }\end{array}$ & 25 & & & & 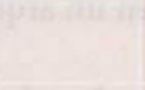 & & \\
\hline $\begin{array}{l}\text { Salida abajo y } \\
\text { rebote balón }\end{array}$ & 1 & 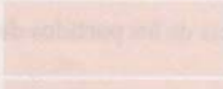 & $2 x^{2}$ & $x=$ & $x_{x}=2$ & & $\sqrt{2}$ \\
\hline Interceptar pie & 1 & & 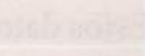 & aine & $\sqrt{3}$ & & \\
\hline Totales & 59 & & 34 & 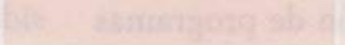 & 37 & & 24 \\
\hline
\end{tabular}

Tabla 3. Grupos de acciones de juego. 
En el primer partido se registraron 25 acciones en el primer tiempo (51\%) y 24 acciones en el segundo tiempo.

(49\%), para un total de 49 acciones que corresponden al $31,8 \%$ de las acciones totales (154). En cuanto a la FC, se tomaron los valores de F1 y F2 y se obtuvo la media.
Se realizaron 16 acciones defensivas (32,6\%), 8 acciones compuestas (16,4\%), 15 acciones ofensivas $(30,6 \%)$ y 10 acciones de habilidad (20,4\%) (gráfica 3).

A partir de la gráfica 3 hasta la 10 se emplean las siguientes convenciones: acciones defensivas $(<)$, acciones compuestas ( $\mathbf{m i n}$ ), acciones ofensivas ( ) y acciones de habilidad ( $\mathbf{\Delta})$.

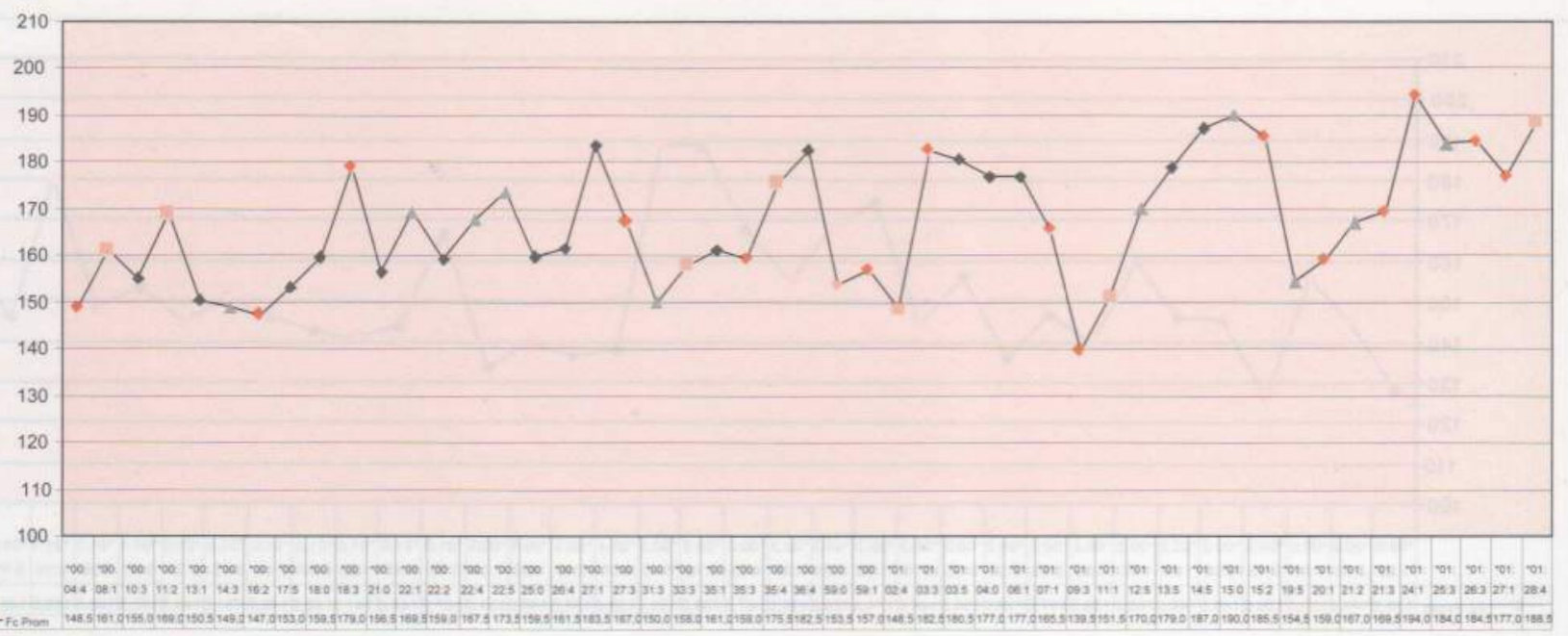

Gráfica 3. Primer partido.

La FC máxima fue 194 lpm, la FC mínima fue 139,5lpm y FC media fue de $167,4 \mathrm{lpm}$.

De las 49 acciones relacionadas con las zonas de intensidad de la frecuencia cardiaca, el arquero realizó 7 acciones en la zona aeróbica ( $-150 \mathrm{lpm}), 31$ acciones en la zona mixta aeróbica/anaeróbica $(151-179 \mathrm{lpm})$ y 11 acciones en la zona anaeróbica $(+180 \mathrm{lpm})$.
En el segundo partido se registraron 14 acciones en el primer tiempo $(46,7 \%)$ y 16 acciones $(53,3 \%)$ en el segundo tiempo, para un total de 30 acciones que corresponden al 19,48\% de las acciones totales.

Se llevaron acabo 11 acciones defensivas (36,6\%), 8 acciones compuestas (26,6\%), 8 acciones ofensivas $(26,6 \%)$ y 3 acciones de habilidad (10,2\%) (gráfica 4).

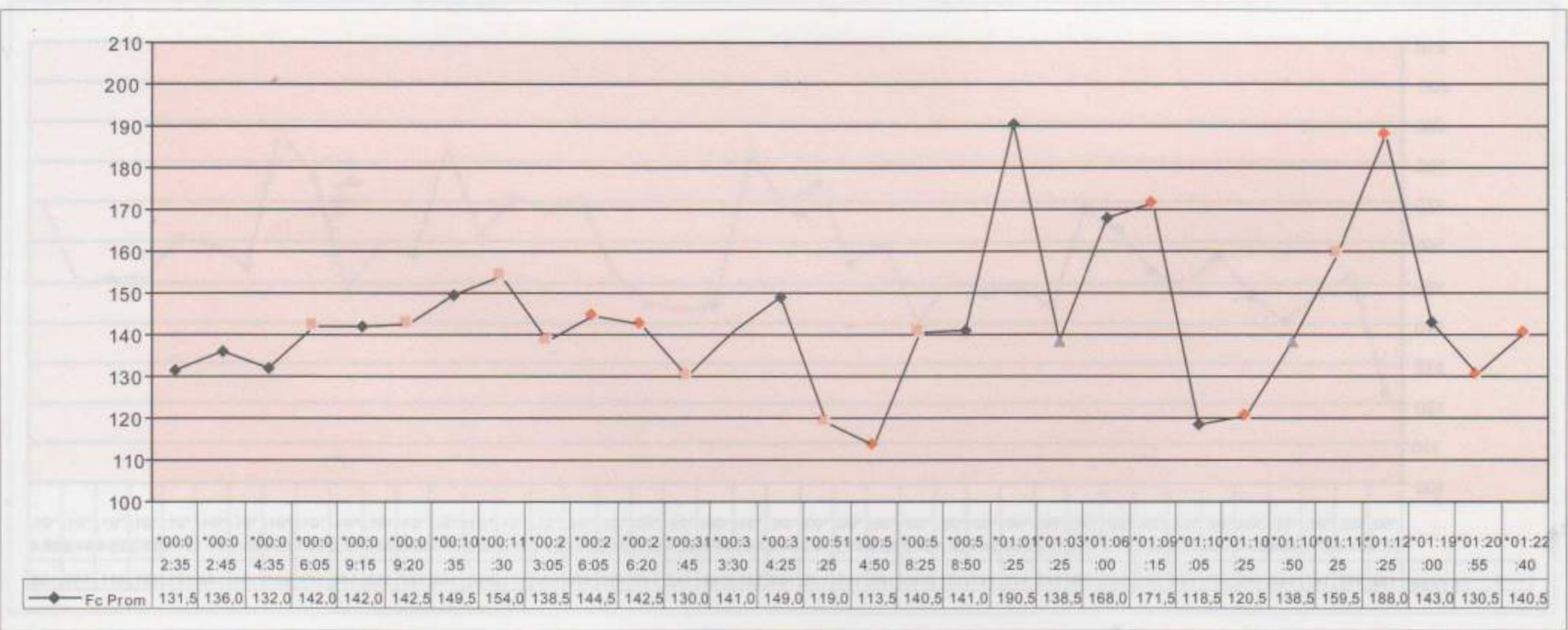

Gráfica 4. Segundo partido. 
La FC máxima fue 190,5 lpm, la FC mínima, 113,5 lpm y el promedio, 151,4 lpm.

De las 30 acciones relacionadas con las zonas de intensidad de la frecuencia cardiaca, el arquero realizó 24 acciones en la zona aeróbica ( $-150 \mathrm{lpm}), 4$ acciones en la zona mixta aeróbica/anaeróbica (151-179 lpm) y 2 acciones en la zona anaeróbica $(+180 \mathrm{lpm})$. En el tercer partido se registraron 18 acciones en el primer tiempo $(52,9 \%)$ y 16 acciones $(47,1 \%)$ en le segundo tiempo para un total de 34 acciones, que corresponden al $22 \%$ de las acciones totales (gráfica 5). Se llevaron a cabo 15 acciones defensivas (44,1\%), 12 acciones compuestas $(35,2 \%), 3$ acciones ofensivas $(8,9 \%)$ y 4 acciones de habilidad (11,8\%).

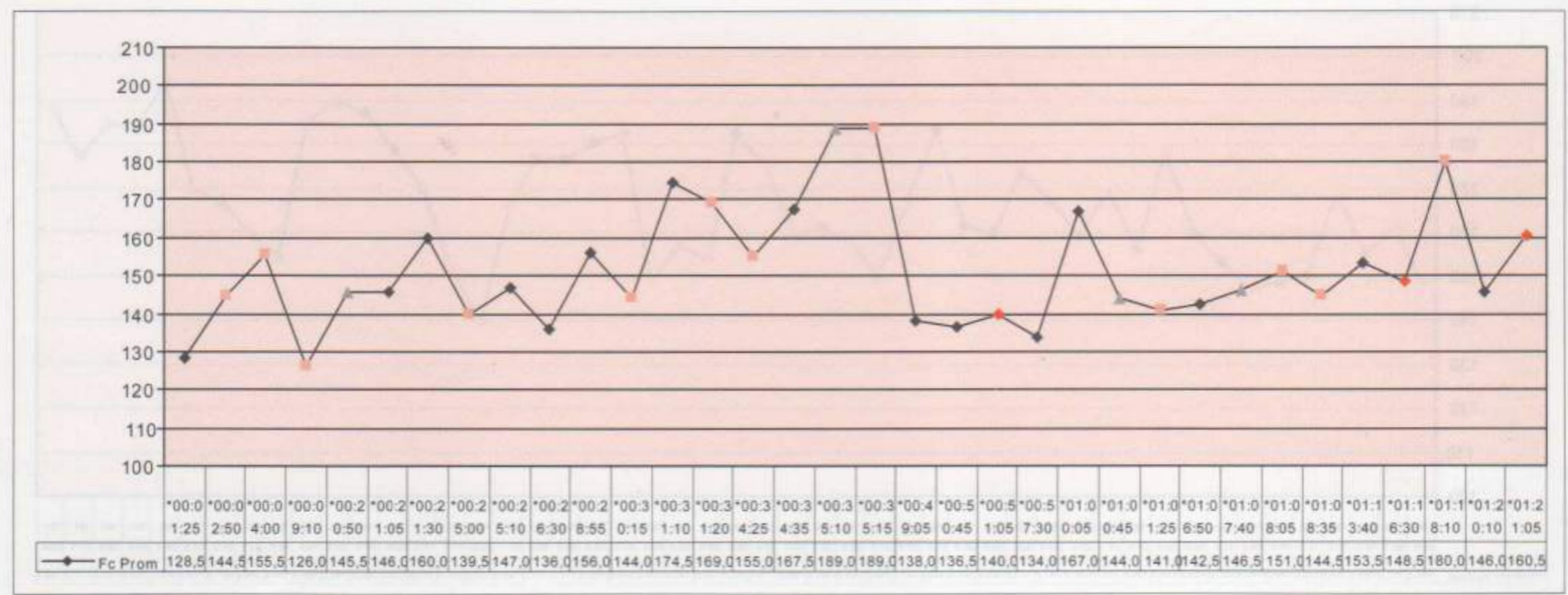

Gráfica 5. Tercer partido.

De las 34 açciones relacionadas con las zonas de intensidad de la frecuencia cardiaca, el arquero realizó 20 acciones en la zona aeróbica ( $-150 \mathrm{lpm}), 11$ acciones en la zona mixta aeróbica/anaeróbica (151-179lpm) y 3 acciones en la zona anaeróbica (+180lpm).
La FC máxima fue 189 lpm, la FC mínima, 126 lpm y el promedio, 151,4 lpm. En el cuarto partido se registraron 20 acciones en el primer tiempo $(48,8 \%)$ y en el segundo tiempo 21 acciones $(51,2 \%)$ para un total de 41 acciones que corresponden al $26,7 \%$ de las acciones totales (gráfica 6).

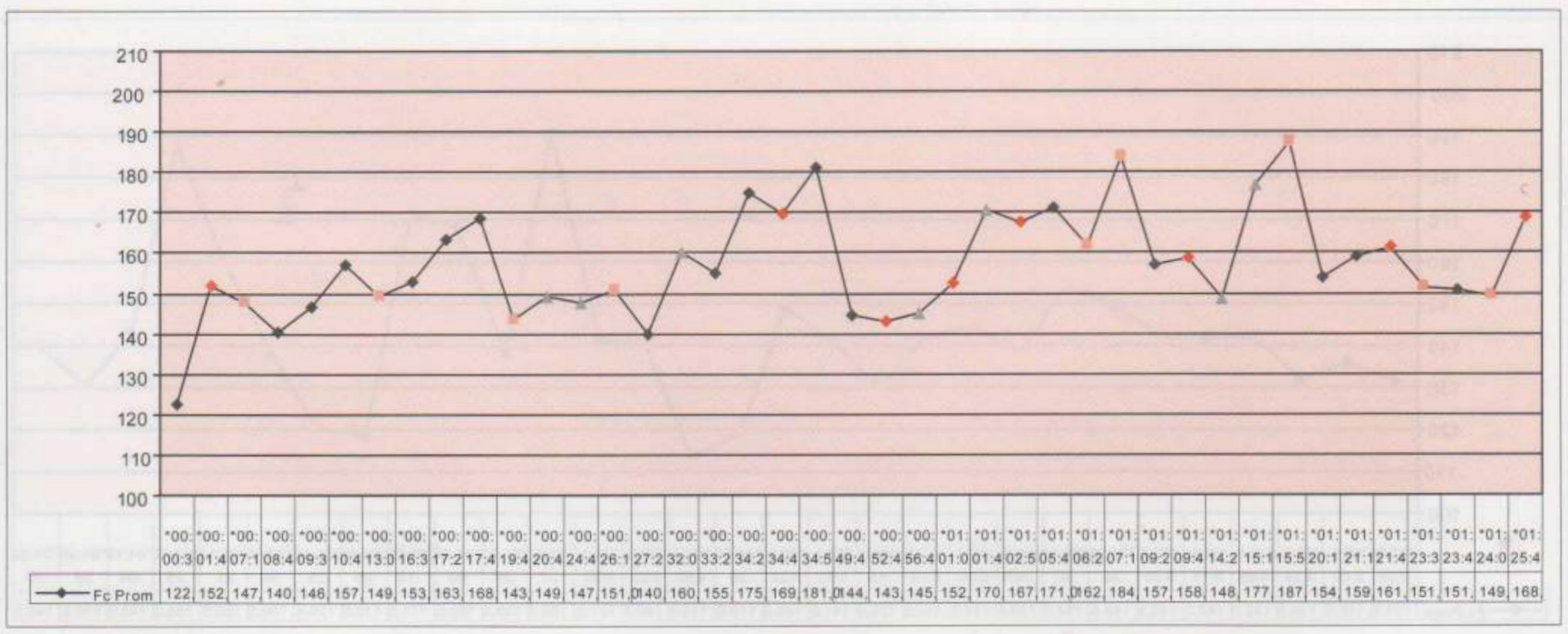

Gráfica 6. Cuarto partido. 
Se llevaron a cabo 17 acciones defensivas $(41,4 \%)$, 9 acciones compuestas (21,9\%), 8 acciones ofensivas $(19,5 \%)$ y 7 acciones de habilidad (17,2\%). La FC máxima, 187,5 lpm, la FC mínima, 122,5 lpm, y el promedio, $157 \mathrm{lpm}$.

De las 30 acciones relacionadas con las zonas de intensidad de la frecuencia cardiaca, el arquero realizó 24 acciones en la zona aeróbica $(-150 \mathrm{lpm}), 4$ acciones en la zona mixta aeróbica/anaeróbica (151-179 lpm) y 2 acciones en la zona anaeróbica $(+180 \mathrm{lpm})$.

De las 41 acciones relacionadas con las zonas de intensidad de la frecuencia cardiaca, el arquero realizó 14 acciones en la zona aeróbica (-150lpm), 24 acciones en la zona mixta aeróbica/anaeróbica (151$179 \mathrm{lpm})$ y 3 acciones en la zona anaeróbica $(+180$ lpm).

\section{Análisis por acción}

\section{Acciones defensivas}

Se realizaron 59 acciones defensivas en los cuatro partidos, que corresponden al $38,4 \%$ de las acciones totales, para un promedio de 14,7 por partido (gráfica 7).

La FC máxima fue 190,5 lpm, la FC mínima, 118,5 y el promedio, fue $155,2 \mathrm{lpm}$.

De las 59 acciones defensivas relacionadas con las zonas de intensidad de la frecuencia cardiaca, el arquero realizó 24 acciones en la zona aeróbica (-150lpm),

29 acciones en la zona mixta aeróbica/anaeróbica (151 - $179 \mathrm{lpm})$ y 6 acciones en la zona anaeróbica $(+180$ lpm).

Se realizaron 8 achiques (14\%), 4 estiradas (7\%), 12 salidas en falso (20\%), 3 salidas y faltas en contra (6\%), 5 salidas con despeje de puño (9\%), 25 salidas y rechazo pie $(42 \%), 1$ salida abajo y rebote de balón (1\%) y una intercepción con el pie (1\%).

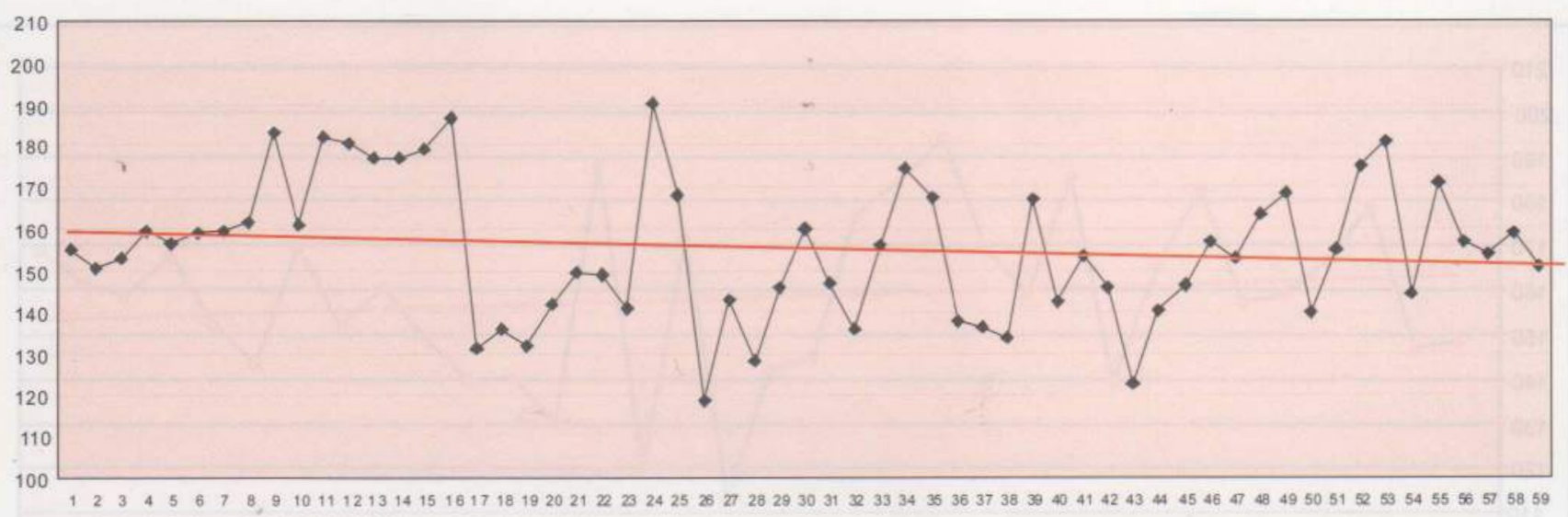

Gráfica 7. Acciones defensivas.

\section{Acciones compuestas}

Se realizaron 37 acciones en los cuatro partidos, que corresponden al $24 \%$ de las acciones totales, para un promedio de 9,3 por partido (gráfica 8 ).

La FC máxima fue 189 lpm, la FC mínima, 119 lpm y el promedio, $153,9 \mathrm{lpm}$.

De las 37 acciones compuestas relacionadas con las zonas de intensidad de la frecuencia cardiaca, el arquero realizó 17 acciones en la zona aeróbica $(-150 \mathrm{lpm}), 15$ acciones en la zona mixta aeróbica/anaeróbica (151-179 lpm) y 5 acciones en la zona anaeróbica $(+180 \mathrm{lpm})$.

Se realizaron 10 recepciones medias y saque de volea $(27 \%), 8$ recepciones bajas y saque de volea $(21 \%), 5$ recepciones con caída y saque $(13,5), 9$ controles con el pie y pase $(25 \%)$ y 5 recepciones altas y saque $(13,5 \%)$. 


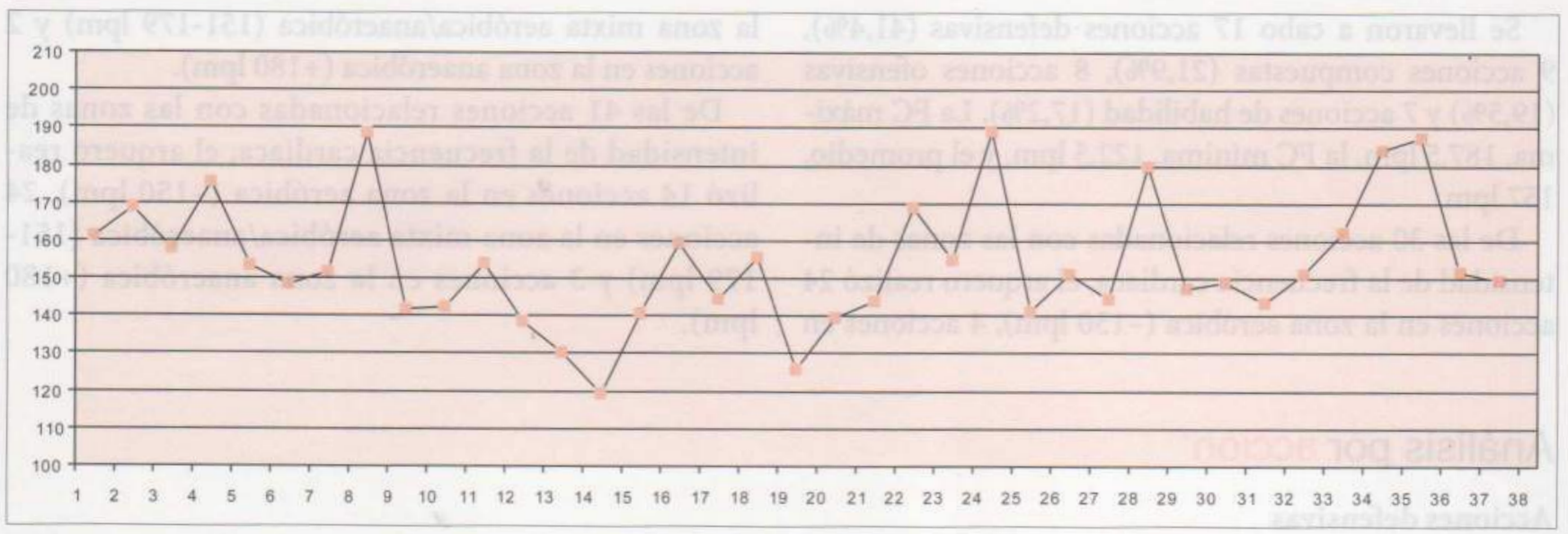

Gráfica 8. Acciones compuestas.

\section{Acciones ofensivas}

Se realizaron 34 acciones en los cuatro partidos, que corresponden al $22 \%$ de las acciones totales, para un promedio de 9,2 por partido (gráfica 9). La FC máxima fue 194 lpm, la FC mínima, 113,5 lpm y el promedio, 158,5lpm. De las 34 acciones ofensivas relacionadas con las zonas de intensidad de la frecuencia cardiaca, el arquero reali-

zó 12 acciones en la zona aeróbica (-150lpm), 17 acciones en la zona mixta aeróbica/anaeróbica (151-179lpm) y 5 acciones en la zona anaeróbica (+180 lpm).

Se realizaron 18 lanzamientos de piso (53\%), 12 saques de piso (35\%) y 4 remates al arco (12\%).

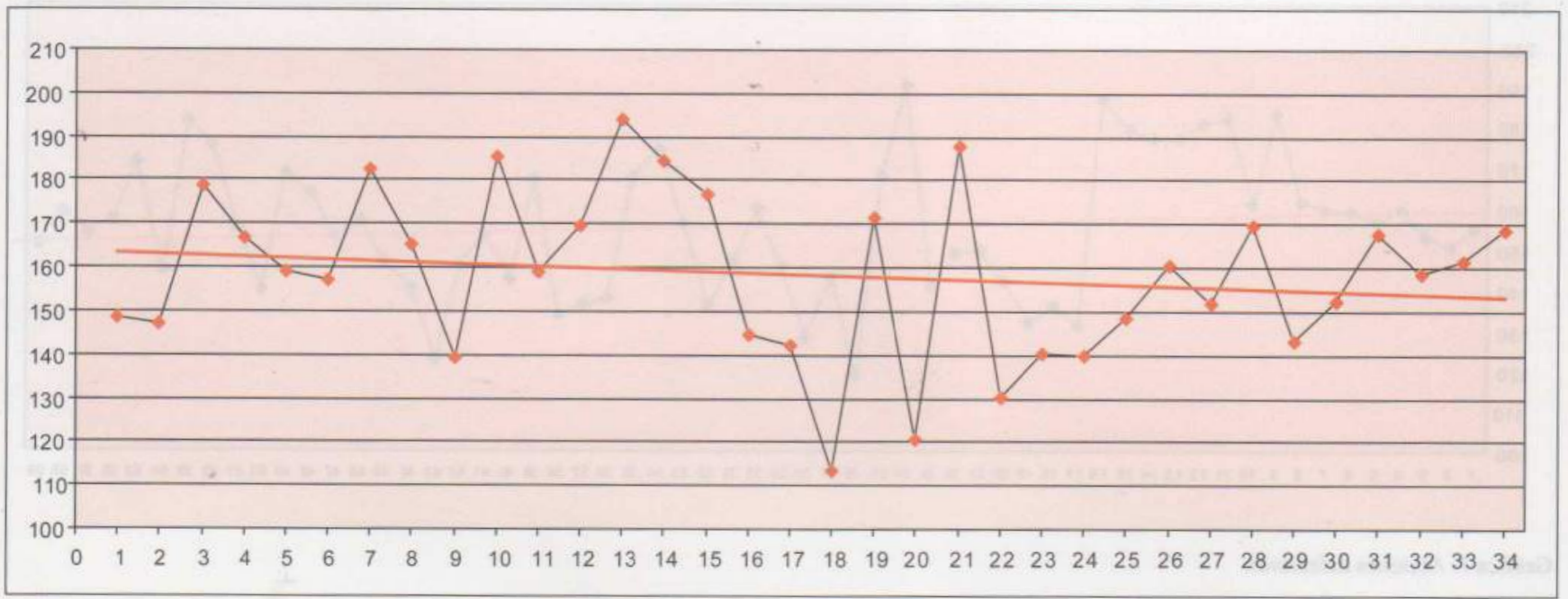

Gráfica 9. Acciones ofensivas.

\section{Habilidades}

Se llevaron a cabo 24 acciones en los cuatro partidos, que corresponden al $16 \%$ de las acciones totales, para un promedio de 6 por partido (gráfica 10). La FC máxima fue 190 lpm, la FC mínima, 138,5lpm y el promedio, $159 \mathrm{lpm}$.

De la 24 acciones de habilidad relacionadas con las zonas de intensidad de la frecuencia cardiaca, el arque- ro realizo 12 acciones en la zona aeróbica $(-150 \mathrm{lpm})$, 9 acciones en la zona mixta aeróbica/anaeróbica (151 - $179 \mathrm{lpm})$ y 3 acciones en la zona anaeróbica $(+180$ $\mathrm{lpm})$.

Se presentaron 13 situaciones estáticas $(53,5 \%), 8$ desplazamientos laterales $(33,5 \%), 2$ saltos verticales (9\%) y un desplazamiento frontal (4\%). 


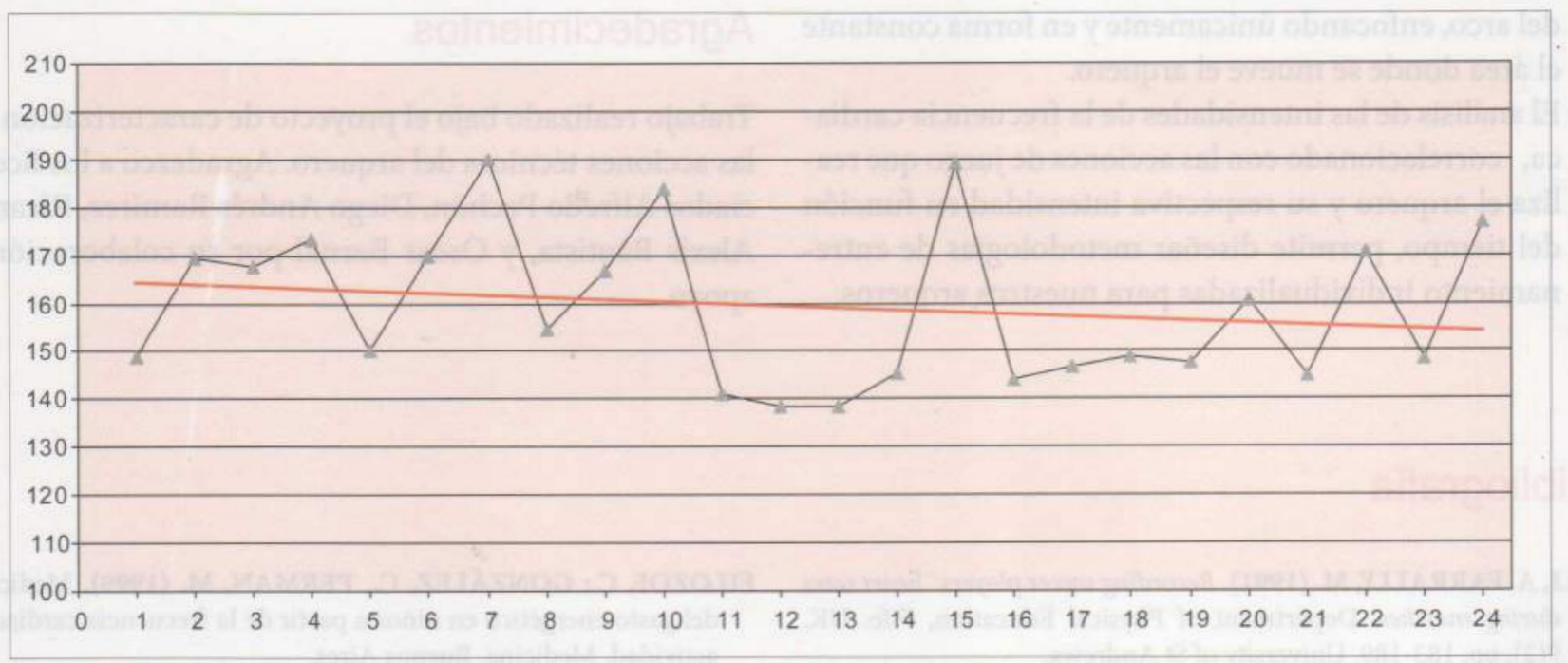

Gráfica 10. Habilidades.

\section{Discusión}

En el entrenamiento deportivo es muy valiosa la frecuencia cardiaca (FC), puesto que es uno de los indicadores que se utilizan cuando se requiere cuantificar la carga de trabajo que un individuo, en nuestro caso el arquero, necesita para la competencia, teniendo en cuenta que éste es uno de los parámetros que indica el nivel de esfuerzo y es muy fácil de tomar. En la actualidad existen instrumentos mucho más sofisticados que permiten medir con mayor precisión y fiabilidad dicho parámetro, pero en los partidos de competencia el reglamento prohíbe utilizar dichos elementos. Desde este punto de vista, fue muy poco lo que pudo realizarse y sólo en cuatro partidos los jueces y el equipo contrario permitieron llevar a cabo la medición.

No existen en este momento investigaciones relacionadas con la frecuencia cardiaca que un arquero desarrolla durante un partido de competencia, por ella valoramos que disponer de 308 registros de FC en un arquero, en cuatro partidos de competencia en la primera ronda clasificatoria, puede ser un inicio para lograr información al respecto.

\section{Conclusiones}

1. La frecuencia cardiaca se comporta con inflexiones ascendentes y descendentes de acuerdo con la característica acíclica propia del fútbol y, en nuestro caso, del arquero.
2. Respecto al porcentaje de la FC máxima teórica, cuando se realizo el estudio el arquero tenía 13 años $(220-13=207 \mathrm{lpm}) ;$ FC máxima teórica $=207 \mathrm{lpm}$ (100\%).

- FC máxima teórica (207lpm), relaciónada con la FC máxima real (186lpm), corresponde al 89,8\%.

- FC máxima teórica (207 lpm), relacionada con la FC promedio real (152 lpm), corresponde al $73,4 \%$.

- FC máxima teórica (207lpm), relacionada con la FC mínima real (107 lpm), corresponde al 51,6\%.

3. De las 154 acciones registradas ( 59 defensivas 37 , compuestas, 34 ofensivas y 24 de habilidad), 65 se llevaron a cabo en la zona aeróbica $(-150 \mathrm{lpm})$, que corresponde al 42,2\%; 70 se realizaron en la zona mixta aeróbica/ anaeróbica (151 - $179 \mathrm{lpm})$, para un $45,5 \%$, y 19 se llevaron acabo en la zona anaeróbica $(+180$ lpm), para un $12,3 \%$.

\section{Recomendaciones}

1. Ampliar la muestra con más arqueros de la categoría para futuras investigaciones similares en Bogotá.

2. Diseñar programas de entrenamiento para el arquero teniendo en cuenta las acciones de juego en general y la referencia de la frecuencia cardiaca en dichas acciones.

3. Para la utilización de los métodos de video recomendamos usar cámaras profesionales ubicadas detrás 
del arco, enfocando únicamente y en forma constante el área donde se mueve el arquero.

4. El análisis de las intensidades de la frecuencia cardiaca, correlacionado con las acciones de juego que realiza el arquero y su respectiva intensidad en función del tiempo, permite diseñar metodologías de entrenamiento individualizadas para nuestros arqueros.

\section{Agradecimientos}

Trabajo realizado bajo el proyecto de caracterización de las acciones técnicas del arquero. Agradezco a los licenciados Alfredo Pachón, Diego Andrés Ramírez, Ricardo Alexis Bautista, y Óscar Bernal por su colaboración y apoyo.

\section{Bibliografía}

ALI, A. FARRALLY, M. (1991). Recording soccer players' heart rates during matches. Department of Physical Education, Fife. UK. 9(2), pp. 183-189. University of St Andrews.

ASTRAD, P.; RODAHL, K. (1985). Fisiologia del trabajo físico. Bases fisiológicas del ejercicio. Buenos Aires. Editorial Médica Panamericana.

BANGSBO, J. (1994). Demandas de energía en el fútbol competitivo. Journal Sports Sciencies. 12, No. especial. Pp. 3-6. England.

BARANDA, P. ORTEGA, E. LLOPIS, L. (2005). Análisis de la acciones defensivas del portero en el fútbol 7. En Revista Apunts. Educación y deportes. Pp. 45-52.

BARBERO, J. ; GRANADA, J. y SOTO, V. (2004). Análisis de la frecuencia cardiaca durante la competición en jugadores profesionales de fútbol sala. En Revista Apunts. pp. 71-78.

BARBERO, J. C. (2003). Análisis cuantitativo de la dimensión temporal durante la competición en fútbol sala. En Motricidad European Journal of Human Movement, X, pp. 143-164.

BERNAL, O., SÁNCHEZ, J. (1995). La frecuencia cardiaca en el periodo competitivo en jugadores mediocampistas de fútbol de rendimiento. Tesis especialización en pedagogía del entrenamiento deportivo. Bogotá. Universidad Pedagógica Nacional.

CAMPOS, J., RAMÓN, V. (2003). Teoría y planificación del entrenamiento deportivo. Paidotribo.

CAPRANICA, L.; TESSITORE, A.; GUIDETTI, L., FIGURA, F. (2001). Heart rate and match analysis in pre-pubescent soccer players. University of Motor Sciences (IUSM), Rome, Italy.

DIRIX, A.; KNUTTGEN, H., TITTLE, K. (1988). Libro Olimpico de la medicina deportiva. Vol. 1. Barcelona. Ediciones, Doyma.
FILOZOF, C.; GONZÁLEZ, C., PERMAN, M. (1999). Medición del gasto energético en niños a partir de la frecuencia cardiaca y actividad. Medicina. Buenos Aires.

FOX, E. (1984). Fisiología del deporte. Buenos Aires: Editorial Médica Panamericana.

GARCIA, J., VALDIVIELSO, M. (1996). Planificación del entrenamiento deportivo. Barcelona: Editorial Gymnos.

GROSGEORGE, B. (1990), Observation et entrainemet en sport collectifs. París: INSEP Public.

LANZA, A. (2003). La respuesta de la frecuencia cardiaca al esfuerzo variable en el juego de fútbol. Año 9, No. 66. Revista Digital, Buenos Aires.

MARTÍNEZ, L. (2001). La Evaluación informatizada en la educación fisica de la ESO. Barcelona. Paidotribo.

McARDIE, W.; KATCH, F., KATCH, V. (1980). Fisiología del ejercicio: energía, nutrición y rendimiento humano. Madrid. Alianza Editorial.

RAMÓN, I. (2004). Progresión didáctica de los contenidos del entrenamiento del portero de fútbol. Wanceulen. Editorial Deportiva, S.L.

TUMILTY, D. (1993). Physiological characteristic of elite soccers players. Sport Medicine. 16 (2). Pp. 80-96. England.

VAUGHAN, N. (2002). Tratado de pediatría. Novena edición. Barcelona. Salvat.

VRETAROS, A. (2003). Comportamento da freqüência cardíaca durante uma sessâo de treino da resistencia específica no tênis de campo: estudo de caso. Año 9, No. 59. Revista Digital. Buenos Aires. 